\title{
Case Report of Necrotizing Autoimmune Myositis With Slightly High Creatinine Phosphokinase
}

\author{
Arshad Yahya ${ }^{\text {a, c }, \text { Kuljeet Singh Anand }}$, Jyoti Garg ${ }^{\mathrm{a}}$, Ravindra Kumar Saran ${ }^{\mathrm{b}}$
}

\begin{abstract}
The idiopathic inflammatory myopathy consists of dermatomyositis (DM), polymyositis (PM), necrotizing autoimmune myositis (NAM) and sporadic inclusion body myositis (sIBM). The specific etiologies of these muscle diseases are not well known and are thought to involve components of the humoral and cellular immune system as well as other nonimmune factors. A case of NAM who was HIV-positive was reported. His muscle biopsy was highly suggestive of NAM but creatinine phosphokinase (CPK) was not favoring the diagnosis. After receiving IVIG pulse therapy, his weakness improved up to that much that he returned back to his work of auto rickshaw driving.
\end{abstract}

Keywords: Dermatomyositis; Polymyositis; Inclusion body myositis; Necrotizing autoimmune myositis; HIV

\section{Introduction}

Inflammatory myopathy (IM) comprises a heterogeneous group of subacute, chronic or sometimes acute acquired muscle diseases, which have in common the presence of moderate to severe muscle weakness and inflammation on muscle biopsy $[1,2]$. Because these diseases represent the largest group of acquired and potentially treatable myopathies both in children and adults, early detection is clinically essential [3]. Based on distinct clinical, immunopathological, histological and prognostic criteria, as well as different degrees of response to therapies, the IM seen in practice can

Manuscript accepted for publication August 13, 2014

${ }^{a}$ Department of Neurology, PGIMER, Dr RML Hospital, New Delhi 110001, India

${ }^{\mathrm{b}}$ Department of Pathology, G B Pant Hospital, New Delhi, India

${ }^{\mathrm{c} C}$ Corresponding Author: Arshad Yahya, Department of Neurology, PGIMER, Dr RML Hospital, New Delhi 110001, India.

Email: drarshadyahya@gmail.com

doi: http://dx.doi.org/10.14740/jnr288w be separated into four distinct subsets: polymyositis (PM), dermatomyositis (DM), necrotizing autoimmune myositis (NAM) and sporadic inclusion body myositis (sIBM) [4, 5]. The disorders have primarily an autoimmune pathogenesis, mediated either by cytotoxic T cells, as in PM and SIBM, by a complement-mediated microangiopathy as in DM, or by macrophages and possibly autoantibodies as in NAM [1-5].

All forms have in common a myopathy characterized by muscle weakness, which usually develops subacutely (weeks to months, as in PM and DM); acutely, even in days, as in NAM; or insidiously over years, as in SIBM, mimicking the progression of a limb girdle muscular dystrophy leading eventually to wheelchair confinement. The patients have difficulty performing tasks requiring the use of proximal muscles, such as getting up from a chair, climbing steps or lifting objects. In advanced disease, respiratory muscles may also be involved, especially in NAM and aggressive forms of DM or PM. In NAM, patients have an acute or subacute onset of severe muscle weakness, serum CK levels in the high thousands [6]. EMG findings of short, small, low-amplitude polyphasic motor unit potentials, fibrillation potentials even at rest, and bizarre high-frequency repetitive discharges support findings of myopathic disease [1, 2]. Anti-SRP antibody has also been reported in NAM. NAM does appear to be influenced by many factors that include malignancy, viral infection like HIV, and statin ingestion [7]. Upregulation of major histocompatibility complex (MHC) class I can be seen in some patients and response to immunomodulatory therapy can occur $[8,9]$.

PM typically demonstrates a lymphocytic infiltration seen mostly within the fascicles (endomysial inflammation), some fiber necrosis, degenerative and regenerative fibers. $\mathrm{CD} 8+\mathrm{T}$ cells are identified in PM and IBM muscle cells expressing MHC class I antigens. In patients with DM, perifascicular atrophy is common. The main features of IBM include endomysial inflammation, vacuolization ("red rimmed vacuoles" on Gomori trichrome stain), and loss of muscle fibers [10]. Large, atrophic, or angulated fibers are also present. Necrosis and macrophage invasion are seen on the NAM biopsies. Biopsy typically lacks a lymphocytic infiltrate; rather there is myocyte swelling and rupture with muscle necrosis. Macrophages are strongly periodic acid Schiff 


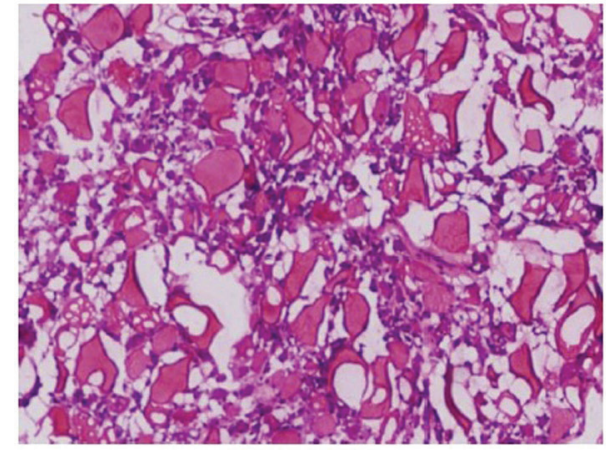

$\mathrm{H} \& \mathrm{E} \times 10$

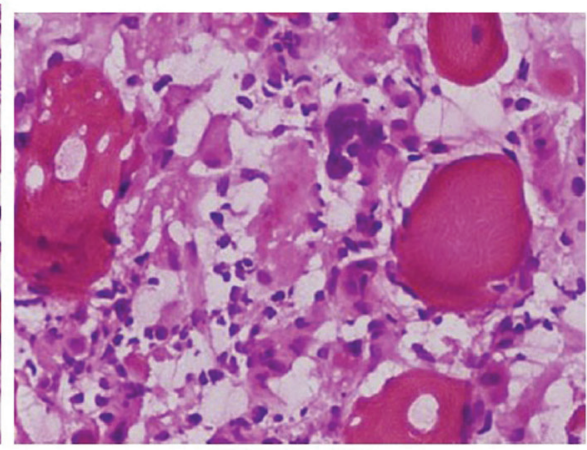

$\mathrm{H} \& \mathrm{E} \times 20$

Figure 1. Loss of fascicular architecture with loss of individual muscle fibers with infiltration of histiocytes and minimal lymphocytes in H\&E staining.

(PAS). They express CD68 and MHC class 1 and MHC class 2 antigens. CD3 T cells, mainly CD8, forming perivascular cuffs are constantly found. Immunohistochemical analysis of complement $\mathrm{C} 5 \mathrm{~b} 9$ shows granular staining of vessels in the endomysium. Electron microscopy observation of the vessels in the endomysium may show tubule reticular particles in endothelial cells of the vessels and destroyed endomysial vessels $[7,11]$.

\section{Case Report}

A 50-year-old non-hypertensive, non-diabetic auto rickshaw driver who had recurrent loose motion, fever and progressive reduction in his weight from the last $4-5$ years presented to us with the complain of gradually progressive weakness of both lower limb in the form of difficulty in standing from squatting position from the last 3 - 4 months along with the pain in the muscles. Gradually he also noted difficulty in climbing stairs. In the mean time due to non-remitting high grade fever he was undergone investigations, in which he was found HIV-positive. His CD4 count was 221, so he was put on HAART in the form of zidovudine $300 \mathrm{mg}$ twice daily, lamivudine $150 \mathrm{mg}$ twice daily and nevirapine $200 \mathrm{mg}$ twice daily. In the febrile period he also noted worsening in his weakness in the form of difficulty in standing from sofa or lower chair, difficulty in elevating the leg in order to sit in crossed leg position, difficulty in turning on the bed, and repeated knee buckling while walking. Due to financial constrains, in spite of weakness he was continuing his driving, but at this point of time he noted weakness in upper limb in the form of difficulty in change of gear and application of clutch. He also noted difficulty in application of hand breaks. Although he also had difficulty in elevating hand above the shoulder but it was minimal. There was no history suggestive of weakness of foot. From the last few weeks he also noted thinning of his anteromedial part of thigh. At the time of presentation he had left his job due to incompetency. There were also no sensory symptoms, cranial nerve involvement, ataxia, spasticity, fasciculations or bladder or bowel involvement. There was also no history of joint pain, skin rashes, hypothyroidism or intake of any hypolipidemic drugs. Family history of muscle weakness was also not present, but history of promiscuous behavior was there.

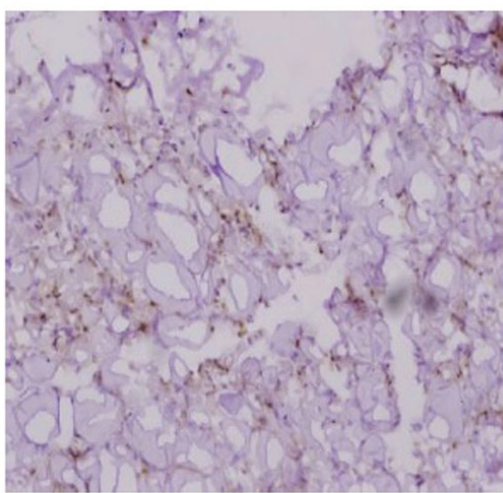

CD68

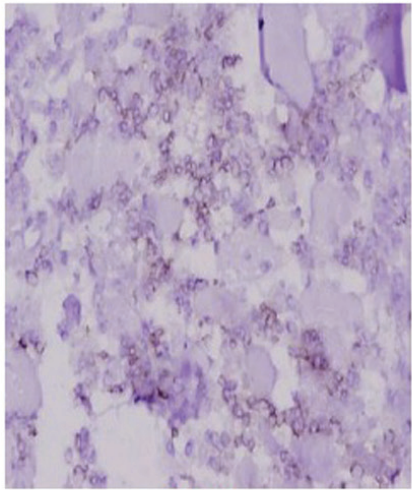

CD3

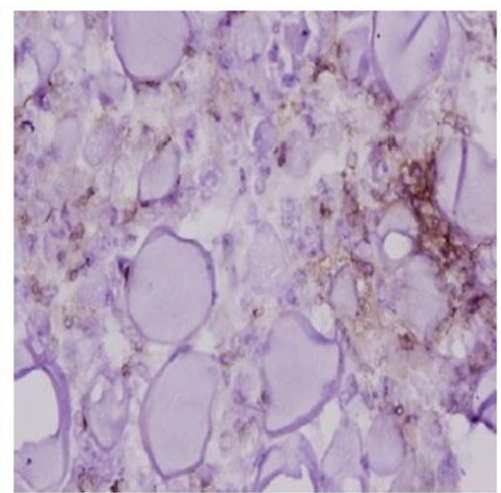

CD8

Figure 2. Immunohistochemistry for CD68 was intensely positive. Focal CD3 and CD8 (T cell) positivity was also noted. 


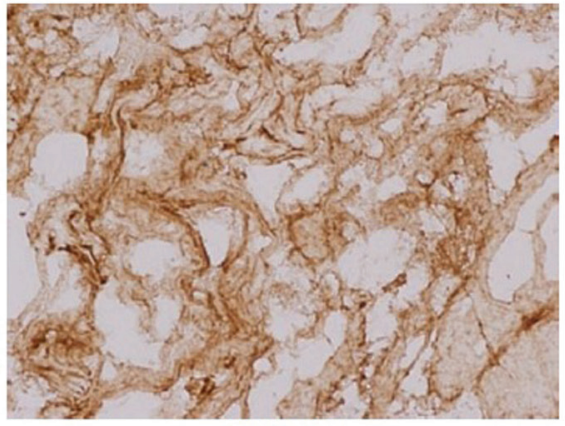

HLA1

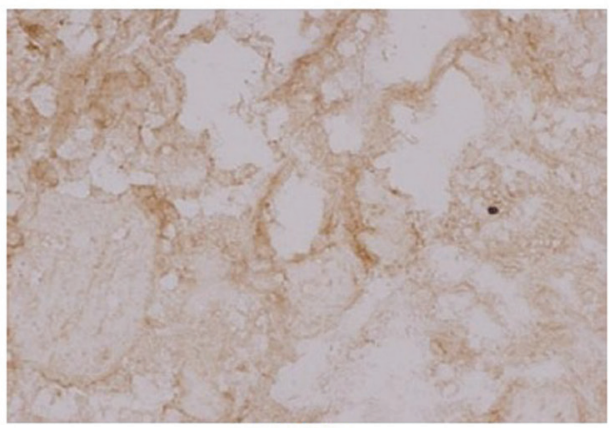

HLA2

Figure 3. In immunohistochemistry sarcolemma also showed overexpression of HLA1 and 2.

On examination, graying of nails was found. Cranial nerve examinations were non-contributory. In the motor examination, atrophy of both sides of muscles of arm and muscles of anteromedial thigh was recorded. There was weakness of neck flexors along with the minimal weakness of muscles of arm. Both hand grips were also weak. In lower limb there was weakness of hip flexor and adductor. Knee flexor was normal but extensors were weak. At the ankle joint weakness was not found. Tendon reflexes both superficial and deep along with sensory system examinations were normal. Both planters were flexor. Other system examination including extrapyramidal and cerebellar system was also normal.

In the investigation his hemoglobin was $8.3 \mathrm{~g} / \mathrm{dL}$, and total leukocyte counts were $5,000 / \mathrm{mm}^{3}$. Kidney function test, bilirubin, total protein, and alkaline phosphatase were normal. Creatinine phosphokinase (CPK) was $453 \mathrm{U} / \mathrm{L}, \mathrm{LDH}$ was $1,308 \mathrm{U} / \mathrm{L}$ and SGOT was $53 \mathrm{U} / \mathrm{L}$. Needle EMG showed low amplitude polyphasic MUAP without any spontaneous activity. Muscle biopsy of vastus lateralis showed loss of fascicular architecture with loss of individual muscle fibers with infiltration of histiocytes and minimal lymphocytes in $\mathrm{H} \& \mathrm{E}$ staining. In many places giant cell formation was also seen. Immunohistochemistry for CD68 was intensely positive. Sarcolemma also showed overexpression of HLA1 and 2. Focal CD3 and CD8 (T cell) positivity was also noted. CD20 was negative (Fig. 1-3). Staining for red ragged fibers was also negative.

On the basis of above mentioned clinical history and examination findings with the characteristic biopsy report, possibility of HIV-associated autoimmune necrotizing myositis was kept. He was put on prednisolone $(1 \mathrm{mg} / \mathrm{kg})$, but within 2 - 3 weeks he presented to with high-grade fever and productive cough. Investigation and clinical findings were suggestive of pneumonia. Steroid was stopped and an intravenous antibiotic was given. For IM, he was kept on pulse IVIG therapy $(2 \mathrm{~g} / \mathrm{kg})$ once every month. He has already received three pulses of IVIG, and is independent and very much competent for his job of driving.

\section{Discussion}

IMs constitute a group of diseases characterized by specifically affecting striated muscle and by their inflammatory nature $[12,13]$. Dermatomyositis is a complement-mediated microangiopathy leading to destruction of capillaries, hypoperfusion and inflammatory cell stress on the perifascicular regions. NAM is an increasingly documented subacute myopathy triggered by statins, viral infections, cancer or autoimmunity with macrophages as the final effector cells causing fiber injury. In PM and sIBM cytotoxic CD8-positive T cells clonally expand in situ and invade major histocompatibility-I-expressing muscle fibers [5].

All forms of IM have in common a myopathy characterized by predominant proximal muscle weakness except IBM. NAM can present acutely or subacutely. NAM can be associated with many factors that include malignancy, viral infection like HIV, and statin ingestion. Necrosis and macrophage invasion are seen on the NAM biopsies. Biopsy typically lacks a lymphocytic infiltrate; rather there is myocyte swelling and rupture with muscle necrosis. Macrophages are characteristically present which are strongly PAS-positive. They express CD68 and MHC class 1 and MHC class 2 antigens. CD3 $\mathrm{T}$ cells, mainly CD8, forming perivascular cuffs are frequently found [1-5]. CPK are usually found in higher ranges, i.e. in thousands. In our case, CPK was slightly higher than normal. On the basis of earlier studies it has been argued that normal ranges for $\mathrm{CK}$ are dependent upon gender, exercise, and ethnicity and are necessarily broad. It is possible for a patient to have muscle injury with a result that is within the reference range. Therefore, some investigators advocate serial testing relative to baseline levels as a more effective method for detecting mild increases in CK. Over the last few years several cases of HIV-associated PM were reported in whom the measured serum CPK was only marginally elevated or even normal. In another study serum CPK values were significantly lower in PM associated with HIV than autoimmune PM cases in the same period of time $[13,14]$. 


\section{Conclusion}

HIV-associated IM may have lesser level of CPK than those without HIV, but the finding should be confirmed by another study with more number of patients.

\section{Conflict of Interest}

No.

\section{References}

1. Dalakas MC. Polymyositis, dermatomyositis, and inclusion-body myositis. N Engl J Med. 1991;325:14871498.

2. Dalakas MC, Hohlfeld R. Polymyositis and dermatomyositis. Lancet. 2003;362(9388):971-982.

3. Mastaglia FL, Phillips BA. Idiopathic inflammatory myopathies: epidemiology, classification, and diagnostic criteria. Rheum Dis Clin North Am. 2002;28(4):723-741.

4. Dalakas MC. Inflammatory muscle diseases: a critical review on pathogenesis and therapies. Curr Opin Pharmacol. 2010;10(3):346-352.

5. Schmidt J, Dalakas MC. Pathomechanisms of inflammatory myopathies: recent advances and implications for diagnosis and therapies. Expert Opin Med Diagn.
2010;4(3):241-250.

6. Dalakas MC. Review: An update on inflammatory and autoimmune myopathies. Neuropathol Appl Neurobiol. 2011;37(3):226-242.

7. Christofer-Stine L, Casciola-Rosen LA, Hong G, Chung T, Corse AM, Mammen AL. A novel autoantibody recognizing 200 and $100 \mathrm{kDa}$ proteins is associated with an immune-mediated necrotizing myopathy. Arthr Rheum. 2010;62:2757-2766.

8. Needham M, Fabian V, Knezevic W, Panegyres P, Zilko P, Mastaglia FL. Progressive myopathy with up-regulation of MHC-I associated with statin therapy. Neuromuscul Disord. 2007;17(2):194-200.

9. Dalakas MC. Toxic and drug-induced myopathies. J Neurol Neurosurg Psychiatry. 2009;80(8):832-838.

10. Castro C, Gourley M. Diagnosis and treatment of inflammatory myopathy: issues and management. Ther Adv Musculoskelet Dis. 2012;4(2):111-120.

11. Miller T, Al-Lozi MT, Lopate G, Pestronk A. Myopathy with antibodies to the signal recognition particle: clinical and pathological features. J Neurol Neurosurg Psychiatry. 2002;73(4):420-428.

12. Bohan A, Peter JB. Polymyositis and dermatomyositis (first of two parts). N Engl J Med. 1975;292(7):344-347.

13. Callen JP. Dermatomyositis. Lancet. 2000;355(9197):5357.

14. Heckmann JM, Pillay K, Hearn AP, Kenyon C. Polymyositis in African HIV-infected subjects. Neuromuscul Disord. 2010;20(11):735-739. 